\title{
Mimicking Phosphorylation at Serine 87 Inhibits the Aggregation of Human $\alpha$-Synuclein and Protects against Its Toxicity in a Rat Model of Parkinson's Disease
}

\author{
Abid Oueslati, ${ }^{1}$ Katerina E. Paleologou, ${ }^{1}$ Bernard L. Schneider, ${ }^{2}$ Patrick Aebischer, ${ }^{2}$ and Hilal A. Lashuel ${ }^{1}$ \\ ${ }^{1}$ Laboratory of Molecular and Chemical Biology of Neurodegeneration and ${ }^{2}$ Neurodegenerative Disease Laboratory, Brain Mind Institute, School of Life \\ Sciences, Ecole Polytechnique Fédérale de Lausanne (EPFL), CH-1015 Lausanne, Switzerland
}

\begin{abstract}
Several lines of evidence suggest that phosphorylation of $\alpha$-synuclein ( $\alpha$-syn) at S87 or S129 may play an important role in regulating its aggregation, fibrillogenesis, Lewy body formation, and neurotoxicity in vivo. However, whether phosphorylation at these residues enhances or protects against $\alpha$-syn toxicity in vivo remains unknown. In this study, we investigated the cellular and behavioral effect of overexpression of wild-type (WT), S87A, and S87E $\alpha$-syn to block or to mimic S87 phosphorylation, respectively, in the substantia nigra of Wistar rats using recombinant adeno-associated vectors. Our results revealed that WT and S87A overexpression induced $\alpha$-syn aggregation, loss of dopaminergic neurons, and fiber pathology. These neuropathological effects correlated well with the induction of hemi-parkinsonian motor symptoms. Strikingly, overexpression of the phosphomimic mutant S87E did not show any toxic effect on dopaminergic neurons and resulted in significantly less $\alpha$-syn aggregates, dystrophic fibers, and motor impairment. Together, our data demonstrate, for the first time, that mimicking phosphorylation at S87 inhibits $\alpha$-syn aggregation and protects against $\alpha$-syn-induced toxicity in vivo, suggesting that phosphorylation at this residue would play an important role in controlling $\alpha$-syn neuropathology. In addition, our results provide strong evidence for a direct correlation between $\alpha$-syn-induced neurotoxicity, fiber pathology, and motor impairment and the extent of $\alpha$-syn aggregation in vivo, suggesting that lowering $\alpha$-syn levels and/or blocking its aggregation are viable therapeutic strategies for the treatment of Parkinson's disease and related synucleinopathies.
\end{abstract}

\section{Introduction}

Increasing evidence suggests that phosphorylation at S87 and S129 may play an important role in regulating $\alpha$-synuclein $(\alpha$ syn) aggregation, fibrillogenesis, Lewy body (LB) formation, and neurotoxicity in vivo (Fujiwara et al., 2002; Anderson et al., 2006; Oueslati et al., 2010; Paleologou et al., 2010). Studies in cell cultures (Okochi et al., 2000; Kim et al., 2006) and animal models of Parkinson's disease (PD) (Fournier et al., 2009; Paleologou et al., 2010) have revealed that $\alpha$-syn is constitutively phosphorylated at S87 (pS87) and S129 (pS129). The levels of pS87 and pS129 were shown to be significantly higher in the brains of patients with PD, Alzheimer's disease (AD), Lewy body disease (LBD), and multiple system atrophy (MSA) and in the corresponding transgenic (Tg) mouse models (Fujiwara et al., 2002; Anderson et al., 2006; Paleologou et al., 2010). Recently, Copeland et al. reported the detection of pS87 in the human CSF and plasma in PD patients (Copeland et al., 2011).

\footnotetext{
Received July 25, 2011; revised Nov. 14, 2011; accepted Dec. 21, 2011.

Author contributions: A.O., B.L.S., K.E.P., P.A., and H.A.L. designed research; A.0. performed research; A.O., K.E.P., B.L.S., P.A., and H.A.L. analyzed data; A.O. and H.A.L. wrote the paper.

This work was supported by EPFL, grants from the Swiss National Science Foundation (31003A_120653) and the Michael J. Fox Foundation, and a Marie Curie post-doctoral fellowship (A. O.). We thank Dr. Yves Sagot for critical review of the manuscript and Prof. Darren Moore for the helpful discussions.

A for-profit corporation, Merck-Serono S.A., partly supported this work.

Correspondence should be addressed to Hilal A. Lashuel at the above address. E-mail: hilal.lashuel@epfl.ch.

DOI:10.1523/JNEUROSCI.3784-11.2012

Copyright $\odot 2012$ the authors $\quad 0270-6474 / 12 / 321536-09 \$ 15.00 / 0$
}

However, whether phosphorylation enhances or protects against $\alpha$-syn toxicity remains unknown. Although the effect of phosphorylation at S129 has been investigated in rat (Gorbatyuk et al., 2008; Azeredo da Silveira et al., 2009; McFarland et al., 2009) and fly models of PD (Chen and Feany, 2005), the role of S87 phosphorylation in $\alpha$-syn fibrillogenesis, LB formation and neurotoxicity in vivo remains unexplored. A recent study by Khandelwal et al. (2010) demonstrated that overexpression of parkin protects against $\alpha$-syn-induced toxicity by reducing the levels of both pS87 and pS129 in vivo, suggesting that phosphorylation at these residues is a pathological event.

S87 lies within the highly hydrophobic non-amyloid component (NAC) region, which has been shown to play a critical role in mediating $\alpha$-syn aggregation and fibrillogenesis in vitro (Uéda et al., 1993; El-Agnaf et al., 1998; Giasson et al., 2001) and in cell culture (Luk et al., 2009). Recent studies from our group and others have demonstrated that phosphorylation at $\mathrm{S} 87$ or $\mathrm{S} \rightarrow$ E substitution (S87E) inhibits $\alpha$-syn fibrillization in vitro (Waxman and Giasson, 2008; Paleologou et al., 2010), which suggest that phosphomimicking mutations may reproduce the effect of phosphorylation. Furthermore, the S87A mutant, which was designed to block phosphorylation, exhibited a similar structure and similar membrane binding and aggregation properties as the wild-type (WT) protein. Together, these findings provide a strong biophysical basis for the use of the S87E and S87A mutants to elucidate the role of phosphorylation at $\mathrm{S} 87$ in vivo. 
Toward this goal, we engineered and stereotaxically injected recombinant adeno-associated vectors (rAAV2/6) overexpressing the WT, S87A or S87E $\alpha$-syn into the rat SNc. We demonstrated that 13 weeks post-injection, the overexpression of WT and S87A $\alpha$-syn induced protein aggregation, dystrophic fiber formation, and a significant loss of dopaminergic neurons in vivo. This neurotoxicity correlated well with the induction of parkinsonian motor impairment as assessed by the cylinder test. Interestingly, the phosphomimic mutant S87E inhibited $\alpha$-syn aggregation and neuronal loss and prevented the formation of dystrophic fibers. These results suggest that $\alpha$-syn phosphorylation at $S 87$ could inhibit $\alpha$-syn toxicity in vivo and demonstrate a direct relationship between $\alpha$-syn aggregation, fiber pathology formation, and neurotoxicity in vivo.

\section{Materials and Methods}

Plasmid construction and production of recombinant AAV2/6 viral vectors. A single nucleotide mutation coding for S87A or S87E was introduced into the cDNA of human $\alpha$-syn using the QuikChange Site-Directed Mutagenesis Kit (Stratagene). Beforehand, the human $\alpha$-syn cDNA had been cloned into the AAV-CMV-MCS backbone (Stratagene). For the S87A variant, the mutagenesis primers were sense 5'-GTGGAGGGAGCAGGGGCCATTGCAGCAGCCACTG-3' and antisense 5'-CAGTGGCTGCTGCAATGGCCCCTGCTCCCTCCAC- $3^{\prime}$. For the S87E variant, the mutagenesis primers were sense 5'-GTGGAGGGAGCAGGGGAAATTGCAGCCACTG-3' and antisense 5'-CAGTGGCTGCAATTTCCCCTGCTCCCTCCAC- $3^{\prime}$. The production and titration of the recombinant pseudotyped AAV2/6 vectors (serotype 2 genome/serotype 6 capsid) were performed as described previously by Azeredo da Silveira et al. (2009). The final titers were $3.88 \times 10^{10}$ transducing units $(\mathrm{TU}) / \mathrm{ml}\left(\alpha\right.$-syn WT), $6.41 \times 10^{10} \mathrm{TU} / \mathrm{ml}$ (S87A), $1.86 \times 10^{11} \mathrm{TU} / \mathrm{ml}$ (S87E), and $5 \times 10^{10} \mathrm{TU} / \mathrm{ml}$ (maxFP-Green).

Stereotaxic injections. All surgical and behavioral procedures were performed in accordance with the Swiss legislation and the European Community council directive (86/609/EEC) for the care and use of laboratory animals.

The injections were performed under xylazine/ketamine anesthesia as described by Paleologou et al. (2010). Male Wistar rats (Charles River Laboratories) weighing $180-200 \mathrm{~g}$ at the time of surgery were placed in the stereotaxic frame (David Kopf Instruments) and received a unilateral intranigral injection of $2 \mu \mathrm{l}$ of viral suspension, which corresponds to a viral load of $2.5 \times 10^{7} \mathrm{TU}$ (maxFP-Green, $n=6$ (immunohistochemistry) and $n=4$ (biochemistry); WT, $n=6$ (immunohistochemistry) and $n=4$ (biochemistry); S87A, $n=5$ (immunohistochemistry) and $n=4$ (biochemistry); S87E, $n=5$ (immunohistochemistry) and $n=4$ (biochemistry)].

Behavioral study: cylinder test. The cylinder test was performed to evaluate the motor impairment induced by $\alpha$-syn overexpression by quantifying the akinesia of the contralateral forelimb as described previously (Schallert et al., 2000; Kirik et al., 2002a). In brief, the rats were placed in a $20 \mathrm{~cm}$ Plexiglas cylinder and videotaped for 10-15 min during their exploratory behavior. A total number of 30 forepaw contacts made on the cylinder wall with the ipsilateral or the contralateral (impaired) forelimbs were scored, and the results were expressed as the percentage of impaired forelimb contacts versus total contacts.

Tissue processing. For immunohistochemistry, the animals were deeply anesthetized and transcardially perfused with $4 \%$ paraformaldehyde (PFA; Fluka, Sigma-Aldrich) 13 weeks post-injection. The brains were removed, post-fixed for $2 \mathrm{~h}$ in PFA, $4 \%$, and then placed in $25 \%$ sucrose. Coronal sections ( $25 \mu \mathrm{m}$ thick) were cut with a microtome (SM2400; Leica) and the slices were stored at $-20^{\circ} \mathrm{C}$ in cryoprotection medium.

For biochemical analysis, the animals were killed using chloral hydrate. The brains were removed and immediately frozen in dry ice. The injected and noninjected hemispheres were cut using a cryostat (300 $\mu \mathrm{m}$ thick), and the midbrains were dissected, collected, and stored at $-80^{\circ} \mathrm{C}$.

Biochemical analysis. Extraction of Tris-soluble fractions from rat midbrains was performed as described previously (Kahle et al., 2001).
For each animal, $50 \mu \mathrm{g}$ of protein was separated in 12\% SDS-PAGE and then transferred to nitrocellulose membrane using a semi-dry blotting system (Bio-Rad). For the detection of $\alpha$-syn aggregates, Tris-soluble fractions from midbrains of 3-4 animals per condition were pooled and precipitated in 10\% trichloroacetic acid (AppliChem). After wash in ethanol and drying, the pellet was resuspended in $1 \times$ loading buffer, electrophoresed in $12 \%$ SDS gel, and transferred to a nitrocellulose membrane using a wet transfer system. Membranes were incubated overnight at $4^{\circ} \mathrm{C}$ with anti-human $\alpha$-syn antibody (1:500; LB509, Zymed Laboratories), anti- $\alpha$-syn (1:1000; BD Bioscience), anti- $\alpha$-syn (1:500; Millipore Bioscience Research Reagents), or anti-GAPDH (1: 20000; Abcam) and then incubated for $1 \mathrm{~h}$ with secondary antibody (goat anti-mouse or anti-rabbit Alexa Fluor IgG). The immunoblots were finally washed and scanned in a Li-COR scanner at a wavelength of 700 or $800 \mathrm{~nm}$.

Immunohistochemistry. Immunohistochemical analysis was performed as described previously by Paleologou et al. (2010). Slices were incubated with primary antibody, anti-tyrosine hydroxylase (1:500; AB152; Millipore), or anti-human $\alpha$-syn (1:500; LB509, Zymed Laboratories) and subsequently incubated with secondary antibodies conjugated to Alexa Fluor-488 or Alexa Fluor-568 (1:1000; Invitrogen) for immunofluorescence or with biotinylated secondary antibody (1:200; Vector Laboratories) for DAB (Pierce) revelation.

Imaging was performed on Leica DMI 4000 microscope (Leica LAS software) equipped with an Olympus AX70 camera. The confocal acquisitions were obtained on Leica DMR XA2 upright confocal microscope (LCS 2 software).

Proteinase $\mathrm{K}$ treatment. Proteinase $\mathrm{K}(\mathrm{PrK})$ treatment was performed according to a protocol described previously by Azeredo da Silveira et al. (2009). After PrK treatment, slices were incubated with primary antibody against human $\alpha$-syn (LB509, Zymed; 1:500) and then revealed with DAB (Pierce), and slides were mounted on glass coverslips.

Unbiased stereologic estimation of dopaminergic neurons in the SNc. The nigral neuronal loss was estimated using unbiased stereology according to the optical fractionator principle described by West et al. (West, 1999). Briefly, the number of TH-immunoreactive neurons and cresyl violet-positive cells was determined every sixth section covering the entire SNc. The analysis was performed using MBF Stereo Investigator software (version 8.0, MBF Bioscience). The parameters used for the stereological analysis were as follows: grid size, $200 \times 180 \mu \mathrm{m}$; counting frame, $75 \times 75 \mu \mathrm{m}$; and $2 \mu \mathrm{m}$ guard zones. The coefficient of error was $<0.1$.

Statistical analysis. Statistical analysis was performed using one-way ANOVA followed by the Sheffé test. Separate analyses were performed between the injected and noninjected side for each group (maxFP-Green, Syn WT, S87A, and S87E). $p<0.05$ was required for rejection of the null hypothesis.

\section{Results}

To investigate the effect of phosphorylation at S87 on $\alpha$-syn aggregation and toxicity in vivo, we used an rAAV2/6 viral system for gene delivery to overexpress human $\alpha$-syn WT or $\alpha$-syn mutants containing substitutions of S87 with alanine (S87A) or glutamate (S87E) to block or to mimic phosphorylation, respectively (Paleologou et al., 2010). To dissociate the effect of the viral injection and the effect of transgene overexpression, we used a control vector overexpressing the fluorescent protein maxFP-Green (Baens et al., 2006) (data not shown).

\section{Human $\alpha$-syn and S87 mutants are expressed throughout the nigrostriatal pathway}

To evaluate the extent of the area that was infected with rAAV in the injected midbrain, we used LB509, a specific antibody against human $\alpha$-syn (Jakes et al., 1999). The staining showed transgenes expression in the entire SNc and revealed a large number of cell bodies expressing human $\alpha$-syn (Fig. $1 A$ ). No $\alpha$-syn immunore- 
A
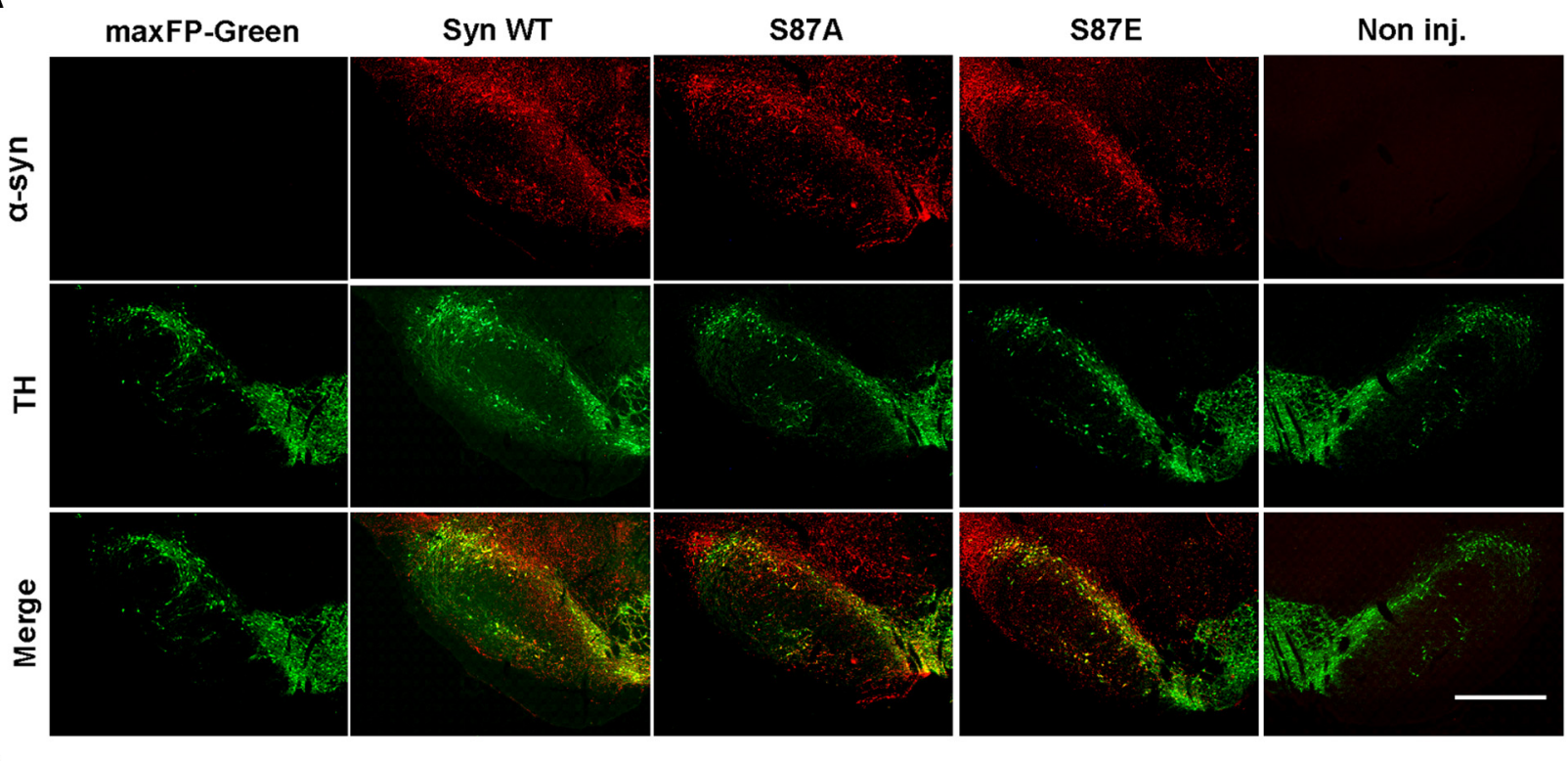

B

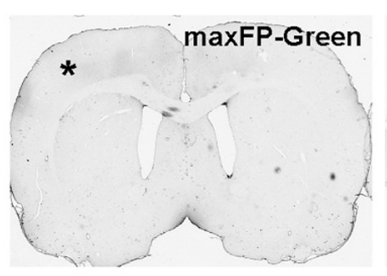

C
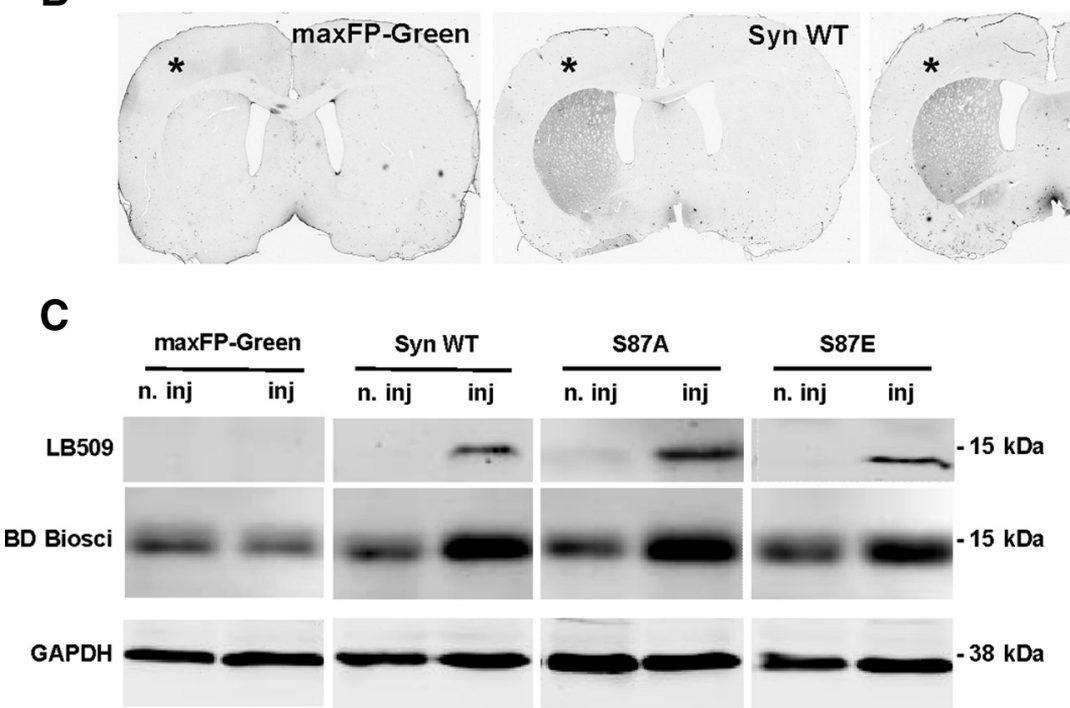
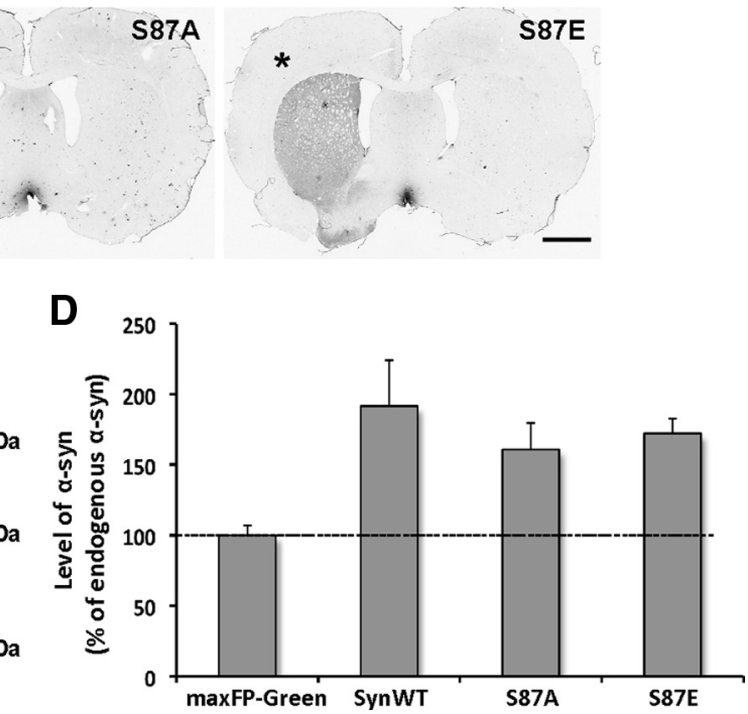

Figure 1. Expression of human $\alpha$-syn and S87 mutants in the nigrostriatal pathway. A, Photomicrographs showing the expression of human $\alpha$-syn variants (red) and dopaminergic neurons (green) in the midbrain. The merge demonstrates that human $\alpha$-syn localizes in the majority of the dopaminergic neurons in the SNc. No human $\alpha$-syn signal was observed in the maxFP-Greeninjected or noninjected sides. Scale bar, $500 \mu \mathrm{m}$. B , Photomicrographs showing the expression of human $\alpha$-syn in the striatal dopaminergic terminals ( ${ }^{*}$, injected side). Scale bar, 2 mm. $\boldsymbol{C}$, Representative immunoblots illustrating the level of overexpressed human $\alpha$-syn. Fifty micrograms of proteins were electrophoresed on $12 \%$ acrylamide gels and probed using a human-specific antibody. $\alpha$-Syn protein was detected as a single $15 \mathrm{kDa}$ band in the injected (inj) sides; however, no band was detected in the maxFP-Green-injected or noninjected (n. inj) sides. Using the BD Biosciences antibody that recognizes both human and rodent $\alpha$-syn, we could detect endogenous rat $\alpha$-syn in the injected and noninjected sides and an increase in $\alpha$-syn level in the injected sides. $\boldsymbol{D}, 0$ ptical density quantification of human $\alpha$-syn level in the injected midbrain revealed a significant increase in $\alpha$-syn level in the injected sides. Compared to endogenous $\alpha$-syn, the quantification indicated that the mean level of total exogenous $\alpha$-syn was approximately equivalent to that of rat endogenous $\alpha$-syn.

activity was detected in the side injected with the maxFP-Green vector or in the noninjected side (Fig. $1 A$ ). Coimmunostaining with tyrosine hydroxylase $(\mathrm{TH})$, a marker of dopaminergic neurons, showed that the majority of $\mathrm{TH}+$ neurons expressed the transgenes (Fig. 1A). It is important to note that we did not observe any significant difference in the nigral distribution of transgene expression between the different groups.

To assess whether the overexpressed proteins were transported to nerve terminals, we probed for human $\alpha$-syn in the striatum. Immunostaining showed an extensive $\alpha$-syn expression in the ipsilateral striatum (Fig. $1 B$ ), and no difference in the transgenes expression levels in the striatal dopaminergic terminals was observed among the groups.
Transgenes expression levels were confirmed by biochemical analysis of Tris-soluble proteins extracted from the midbrain. Western blot analysis showed a single band at $15 \mathrm{kDa}$ corresponding to human $\alpha$-syn, whereas no signal was detected in the noninjected or in the maxFP-Green-injected midbrains (Fig. $1 C)$. To get an estimate for the level of the overexpressed $\alpha$-syn, we used an antibody that recognizes both human and rodent $\alpha$-syn. Western blot analysis showed a dramatic increase in $\alpha$-syn level in the injected compared to the noninjected side (Fig. 1C). Optical density quantification confirmed $\alpha$-syn overexpression and provided an estimate of the mean total level of exogenous $\alpha$-syn, which was approximately equivalent to the rat endogenous $\alpha$-syn $(\approx 175 \%$ ) (Fig. $1 D)$. 


\section{Human $\alpha$-syn WT and S87A are toxic, but S87E is not}

To evaluate the toxicity effect of overexpressed human $\alpha$-syn WT and S87 mutants on the dopaminergic neurons in the SNc, we used unbiased stereological quantification of $\mathrm{TH}+$ neurons. The comparison of the total number of $\mathrm{TH}+$ neurons, in the injected versus noninjected side did not show any significant difference in maxFP-Green and S87E groups $(p=0.375$ and $p=0.517$, respectively) (Fig. $2 A, B$ ). However, the number of dopaminergic neurons appeared to be significantly reduced after overexpression of $\alpha$-syn WT and S87A ( $p=0.027$ and $p=0.01$, respectively) (Fig. $2 A, B$ ). To evaluate the extent of the degeneration of the dopaminergic neurons between the different experimental conditions, we expressed the data as $\mathrm{TH}+$ neuron loss. Quantification of the neuronal loss revealed a significant difference among all conditions $\left(F_{(3,18)}=15.41, p<0.0001\right)$. Interestingly, compared to the control group (maxFP-Green), we measured a significant neuronal loss $(\approx 30 \%)$ in the $\alpha$-syn WT and S87A groups ( $p=0.0004$ and $p=0.0002$, respectively) (Fig. $2 C$ ). However, no significant neurodegeneration was observed in the S87E group $(p=0.38)$ (Fig. $2 C$ ).

To confirm that the decrease in $\mathrm{TH}+$ neurons number is due to neuronal loss rather than the loss of dopaminergic phenotype, we performed a stereological quantification of cresyl violetpositive $(\mathrm{CV}+)$ cells. The number of $\mathrm{CV}+$ cells was significantly decreased in the SNc injected with viruses overexpressing WT and S87A $\alpha$-syn compared to the noninjected side ( $p=0.045$ and $p=0.02$, respectively) (Fig. $2 D$ ), whereas no significant loss was observed in the maxFP-Green- and S87E-injected groups $(p=$ 0.16 and $p=0.53$, respectively) (Fig. $2 E$ ). Furthermore, the analysis of the percentage in $\mathrm{CV}+$ loss compared to the noninjected side showed a significant difference among all groups $\left(F_{(3,18)}=\right.$ 41.59; $p<0.0001$ ). Compared to the control group (maxFPGreen), the analysis revealed a significant cell loss $(\approx 25 \%)$ after the injection of WT or S87A $\alpha$-syn $(p<0.0001)$, and no significant effect was observed after S87E overexpression. The percentage of CV + cell loss was nearly the same as that of TH+ cell loss, which confirms dopaminergic neuron loss in the injected SNc. These results demonstrate that the overexpression of WT or S87A $\alpha$-syn induces a significant dopaminergic neuron loss, while the phosphomimic S87E mutant does not display any significant toxicity.

To assess whether the neuronal degeneration induces dopaminergic fiber loss in the ipsilateral striatum, we performed TH optical density quantification. The results showed a significant decrease of $\mathrm{TH}$ staining intensity in the injected side compared to the noninjected side in the WT and S87A $\alpha$-syn groups ( $\approx 20 \%$; $p<0.01$ and $p<0.05$, respectively) (Fig. $2 F, G)$, indicating that the neuronal degeneration in the $\mathrm{SNc}$ is associated with a dopaminergic fibers loss in the ipsilateral striatum. However in maxFP-Green and S87E-injected animals, no significant fiber degeneration was observed (Fig. $2 F, G$ ).

\section{Human $\alpha$-syn WT and S87A mutant induce striatal fiber pathology, but the S87E mutant does not}

Neuropathological observations in synucleinopathy-diseased brains (Irizarry et al., 1998; Braak and Braak, 2000; Duda et al., 2000) and animal models of PD (Kirik et al., 2002b; Koprich et al., 2010) describe the presence of striatal dystrophic neurites. To determine whether the overexpression of $\alpha$-syn WT and S87 mutants induces fiber pathology in the striatum, we performed a dopaminergic terminal staining using TH antibody. Strikingly, at high magnification Syn WT and S87A-injected animals showed $\mathrm{TH}+$ enlarged fibers in the dorsolateral part of the striatum (Fig.
3). In contrast, there were fewer dystrophic fibers in the S87E group, and none in the maxFP-Green control group (Fig. 3). This observation demonstrates that only Syn WT and S87A induce striatal fiber pathology.

\section{Human $\alpha$-syn WT and S87A induce hemi-parkinsonian motor symptoms}

To evaluate the effect of human WT, S87A, and S87E $\alpha$-syn overexpression on animal motor performances, we used the cylinder test to assess how utilization of the contralateral forepaw was affected (akinesia) in response to the dopaminergic neuronal loss (Schallert et al., 2000; Kirik et al., 2002a). As illustrated in Figure 4 , before injection and in all our experimental conditions, the animals used their forepaws equally with $\sim 50 \%$ use of each forelimb. Interestingly, 13 weeks post-injection, the animals overexpressing WT and S87A $\alpha$-syn showed a significant reduction in contralateral forepaw use, compared to their pre-injection performances ( $p=0.0013$ and $p=0.003$, respectively). This reduction reflected a forelimb motor asymmetry, which is characteristic of hemi-parkinsonian symptoms. By contrast, no significant differences were observed between the pre-injection and post-injection performances of rats overexpressing maxFPGreen or S87E. This data demonstrates that only the overexpression of WT and S87A $\alpha$-syn induces hemi-parkinsonian motor symptoms.

\section{Human $\alpha$-syn WT and S87A produce more insoluble $\alpha$-syn striatal aggregates than the S87E mutant}

The presence of striatal $\alpha$-syn insoluble aggregates resistant to proteinase $\mathrm{K}(\mathrm{PrK})$ is one of the neuropathological features of PD and related disorders (Neumann et al., 2002). To determine the effect of mutations at S87 on $\alpha$-syn aggregation in vivo, we assessed the presence of $\alpha$-syn-insoluble species in the ipsilateral striatum. As illustrated in Figure 5, WT and S87A groups showed $\alpha$-syn-positive deposits and dystrophic neurites. These deposits were less pronounced in the case of S87E and were completely absent in the maxFP-Green group (Fig. 5A). After digestion with $\operatorname{PrK}$, the $\alpha$-syn-positive deposits and neurites were still observed in the striatum of the Syn WT and S87A-injected animals. However, we observed a significant reduction in $\alpha$-syn staining in the ipsilateral striatum of S87E-injected animals (Fig. 5A). This observation demonstrates that the overexpression of WT and S87A $\alpha$-syn, in contrast to the S87E mutant, correlates with the production of $\alpha$-syn aggregates in the striatal dopaminergic terminals. Moreover, double immunostaining using human $\alpha$-syn antibody and thioflavin $\mathrm{S}$ (ThS), a marker of $\beta$-sheet rich amyloid-like structures, revealed a high degree of colocalization in the dystrophic neurites in Syn WT and S87A-injected animals, suggesting that $\alpha$-syn in the dystrophic fibers forms amyloid-like aggregates (data not shown). To confirm biochemically the formation of $\alpha$-syn aggregation, we extracted and precipitated proteins from the midbrain regions of 3-4 animals per condition. Western blot analysis confirmed that Syn WT and S87A induced the formation of more high molecular $\alpha$-syn species compared with S87E or maxFP-Greeninjected animals (Fig. 5B), suggesting that WT and S87A $\alpha$-syn produce more aggregates. All together, these immunohistological and biochemical data confirm that S87E mutant produces less $\alpha$-syn aggregation in vivo compared to WT and S87A $\alpha$-syn constructs.

\section{Discussion}

$\alpha$-syn phosphorylation at S87 and S129 has been reported to play important roles in regulating $\alpha$-syn interaction with proteins, 
A

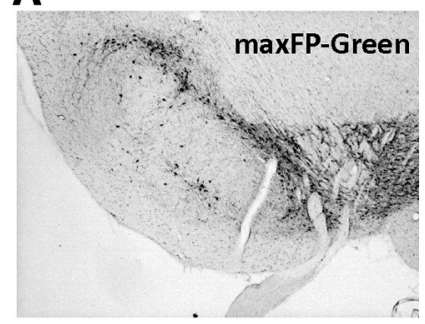

B

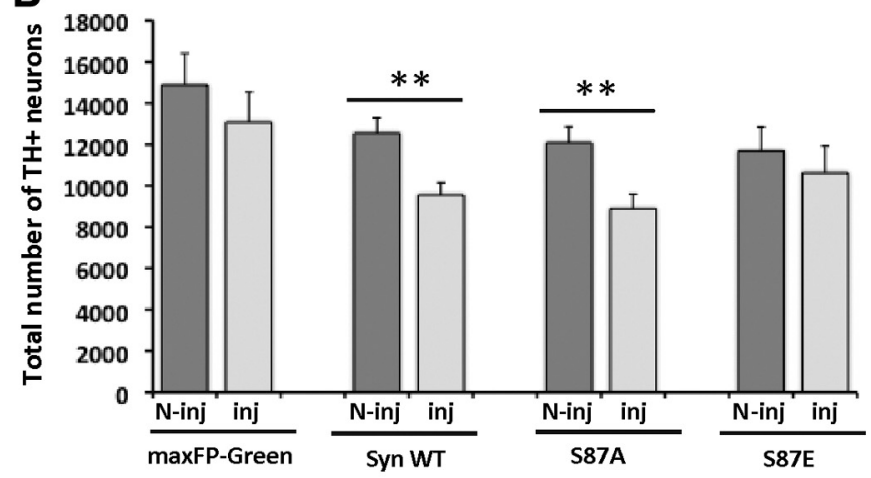

D

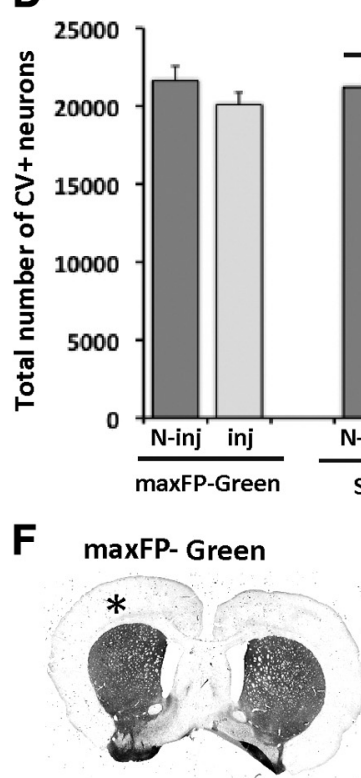

S87A
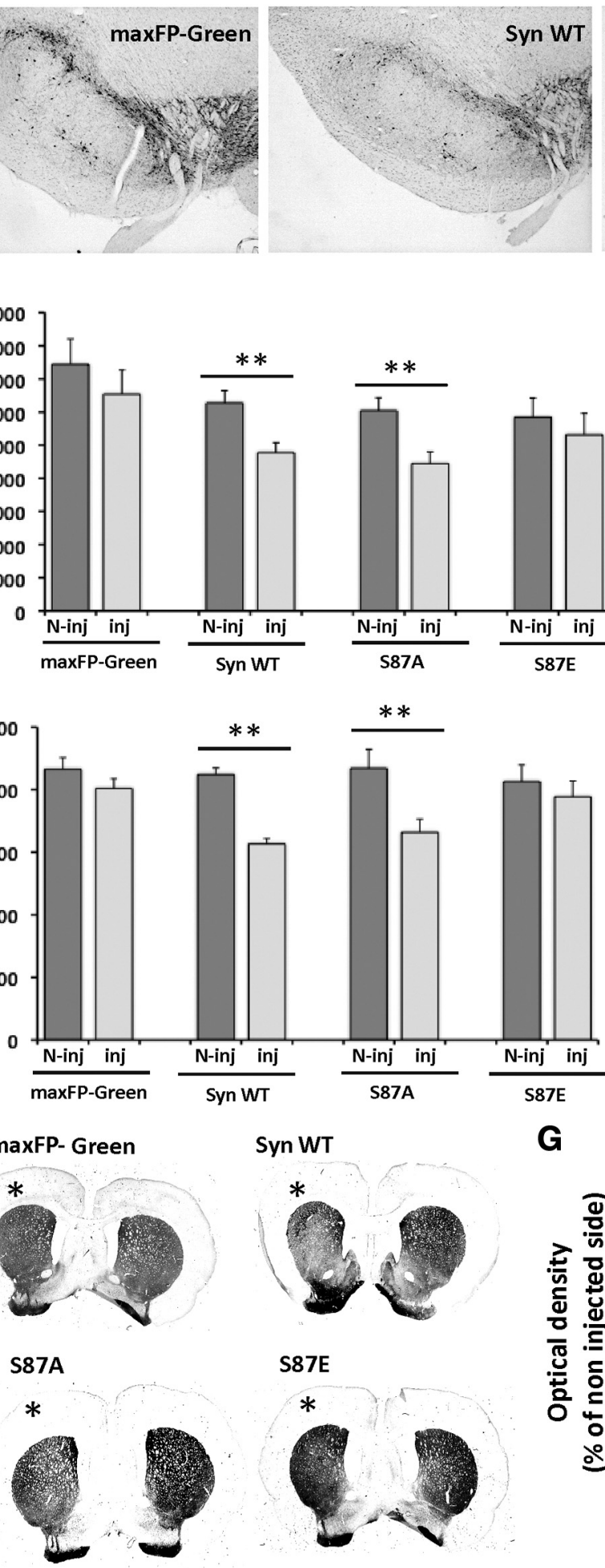

Syn WT

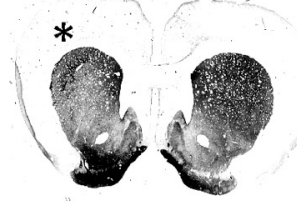

S87E

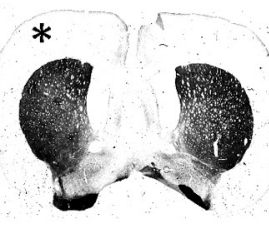

G

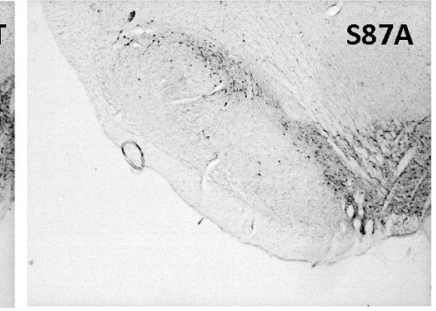

C

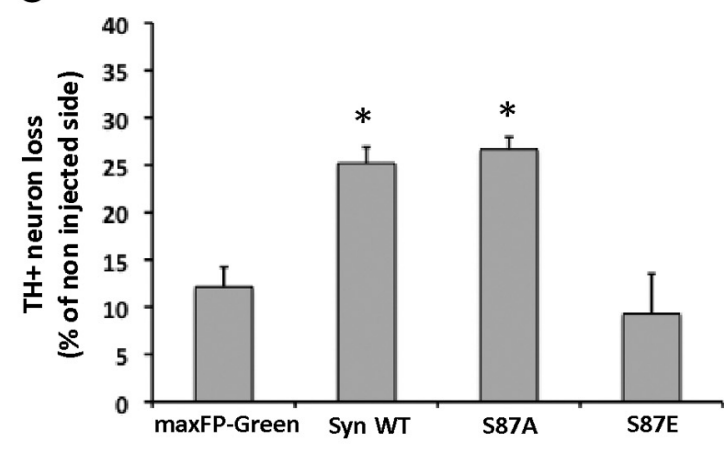

E

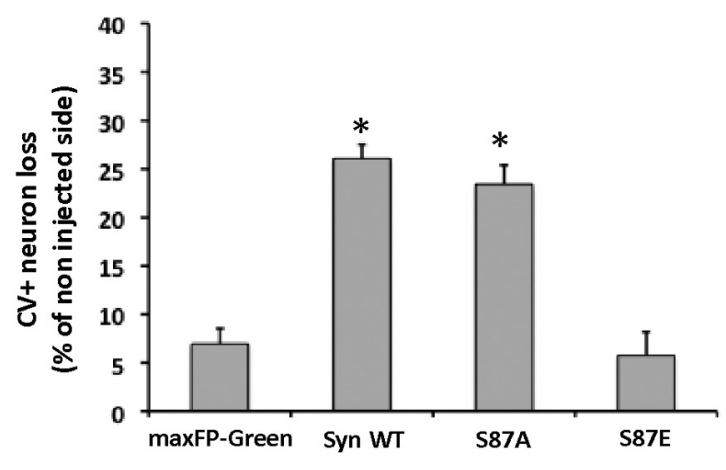

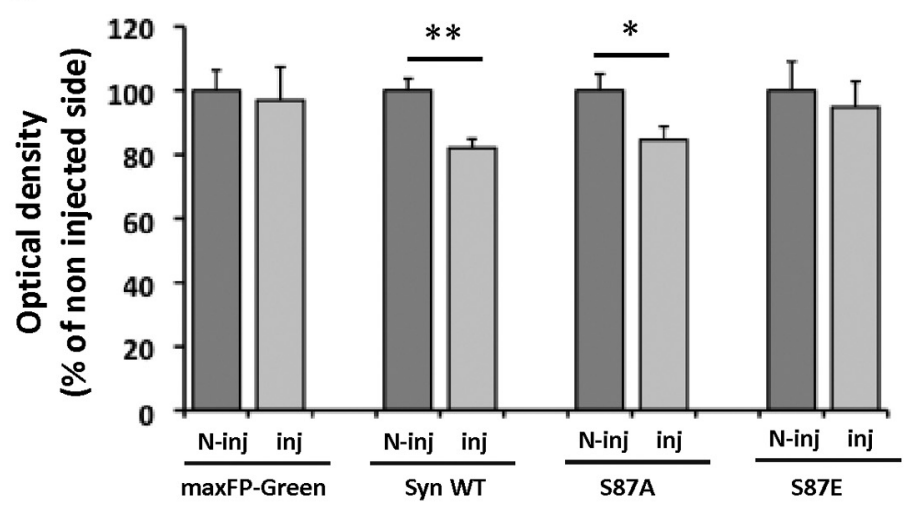

Figure 2. Toxicity of the overexpressed human $\alpha$-syn and S87 mutants in the injected SNc. A, Photomicrographs illustrating the dopaminergic (TH +) neuronal degeneration in the injected SNc. The slices were stained using the anti-TH antibody and showed a reduction in TH signal in the SNc overexpressing human $\alpha$-syn WT and S87A compared to the maxFP-Green and S87E-injected brains. Scale bar, $300 \mu \mathrm{m}$. B, Unbiased stereological quantification of TH + neurons in the injected (Inj) and noninjected (N-Inj) SNc. The comparison with the noninjected side revealed a significant neuronal loss in the SNc overexpressing human syn WT and S87A $\left({ }^{* *} p<0.001\right.$, Sheffé test). However, no significant neuronal loss was observed in the maxFP-Green or S87E-injected brains. C, When compared with the control group (maxFP-Green), the analysis showed a significant toxicity after human $\alpha$-syn WT and S87A overexpression ( ${ }^{*} p<0.05$, Sheffé test), whereas, S87E overexpression appeared to be nontoxic. $\boldsymbol{D}, \boldsymbol{E}$, Stereological quantification of cresyl violet CV + cells in the SNc. The analysis showed a significant decrease in the number of CV + cells in the syn WT and S87A-injected SN compared to the noninjected side $\left({ }^{* *} p<0.001\right.$, Sheffé test) $(\boldsymbol{D})$ and a significant toxicity for syn WT and S87A overexpression compared to the control group (maxFP-Green) $\left({ }^{*} p<0.05\right.$, Sheffé test) $(\boldsymbol{E}) . \boldsymbol{F}, \boldsymbol{G}$, Photomicrographs showing the dopaminergic $(\mathrm{TH}+)$ fiber degeneration in the injected side $\left(^{*}\right)(\boldsymbol{F})$ and optical density quantification revealing a significant decrease of TH staining intensity in the injected side in the Syn WT and S87A groups $\left({ }^{*} p<0.05\right.$, Sheffé test) $(\boldsymbol{G})$. 
maxFP-Green

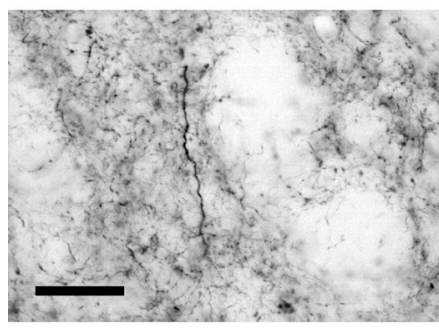

Syn wt

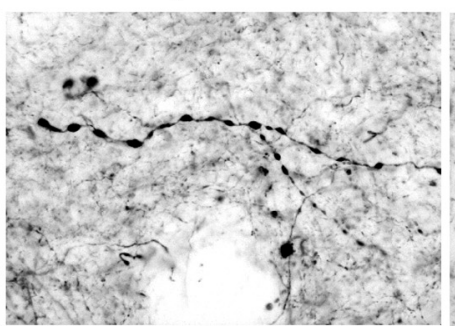

S87A

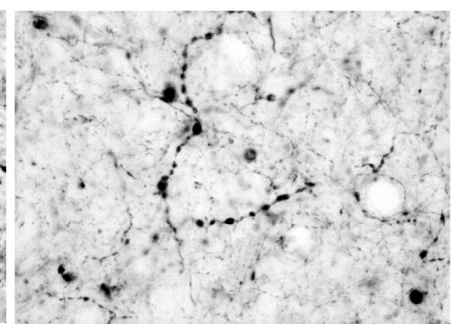

S87E

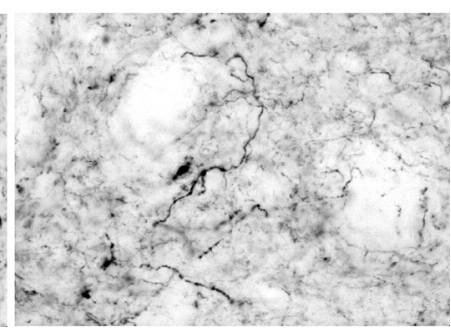

Figure 3. Induction of striatal fiber pathology after the overexpression of syn WT and S87A. At high magnification, TH staining in the ipsilateral striatum revealed the presence of numerous swollen and dystrophic fibers. Strikingly, only rare swollen fibers were observed in the striatum of S87E-injected rats, which suggests that the overexpression of this mutant did not induce fiber pathology. In the control group overexpressing maxFP-Green, the analysis did not show any dystrophic fibers in the ipsilateral striatum. Scale bar, $10 \mu \mathrm{m}$.

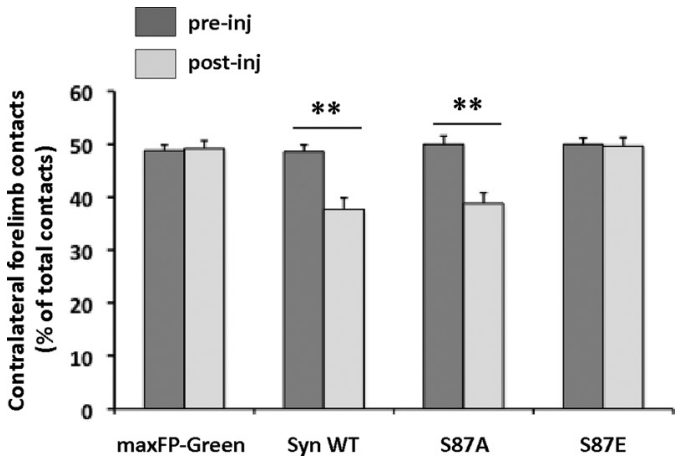

Figure 4. Induction of hemi-parkinsonian motor impairments after the overexpression of human $\alpha$-syn WT and S87 mutants. Assessment of the motor impairment induced by dopaminergic degeneration was performed using the cylinder test. The histograms show that before the injection (pre-inj), the animals in all of the conditions had symmetrical motor activity. Interestingly, 13 weeks post-injection (post-inj) the quantification showed significantly impaired contralateral contacts in the WT and S87A $\alpha$-syn-injected groups $\left({ }^{* *} p<0.001\right.$, Sheffé test), which reflect forelimb use asymmetry. However, the animals injected with viruses overexpressing maxFP-Green or S87E did not show any change in motor performances after the viral injection.

axonal transport (pS129: McFarland et al., 2008; Lou et al., 2010; $\mathrm{Wu}$ et al., 2011), and degradation (pS129: Chau et al., 2009; Machiya et al., 2010), as well as its own aggregation (pS87: Waxman and Giasson, 2008; Paleologou et al., 2010; pS129: Smith et al., 2005, Paleologou et al., 2008) and toxicity (pS129: Sugeno et al., 2008; Kragh et al., 2009). Human $\alpha$-syn within LBs is phosphorylated at S129 and S87, and several groups have reported significant increases in pS87 and pS129 levels in patients with AD, LBD, and MSA (pS129; Fujiwara et al., 2002, Anderson et al., 2006) (pS87; (Paleologou et al., 2010) as well as in Tg mice and rat models of synucleinopathies (pS129: Kahle et al., 2002; Zhou et al., 2008; Khandelwal et al., 2010). However, whether phosphorylation enhances or protects against $\alpha$-syn toxicity remains unclear. Although the effect of phosphorylation at S129 has been investigated in rat (Gorbatyuk et al., 2008; Azeredo da Silveira et al., 2009; McFarland et al., 2009) and fly models of PD (Chen and Feany, 2005), the role of $\alpha$-syn phosphorylation at S87 in $\alpha$-syn fibrillogenesis, LB formation, and neurotoxicity in vivo remains unexplored.

To investigate the effect of phosphorylation at S87, we first considered coexpression of WT $\alpha$-syn with a kinase that phosphorylates the protein efficiently at this residue. Only casein kinase I (CKI) has been shown to reproducibly phosphorylate $\alpha$-syn at S87 in vitro and in cell cultures and to colocalize with pS87 $\alpha$-syn in LBs (Waxman and Giasson 2008; Paleologou et al., 2010). However, CKI phosphorylates $\alpha$-syn efficiently at several other residues, including S129 and to a lesser extent T92, which precludes any specific assessment of the role of S87 phosphorylation (Waxman and Giasson, 2008; Paleologou et al., 2010). Faced with this limitation, we considered the use of the $\alpha$-syn mutants S87E and S87A to mimic or block phosphorylation in vivo as an alternative strategy. In vitro biophysical studies from our group and others (Waxman and Giasson, 2008; Paleologou et al., 2010) have demonstrated that phosphorylation at S87 or substitution of S87 with glutamate (S87E) inhibits $\alpha$-syn oligomerization and fibril formation in vitro, suggesting that phosphomimicking mutations (S87E/D) may reproduce the effect of phosphorylation in vivo. We also showed that the S87A mutant exhibits similar biophysical and aggregation properties as WT $\alpha$-syn (Paleologou et al., 2010). These findings provided a strong biophysical basis for the use of the S87E and S87A mutants to investigate the effect of S87 phosphorylation in vivo.

\section{Overexpression of WT and S87A, but not the S87E mutant,} induces the loss of dopaminergic neurons, fiber pathology, and motor deficit in a rat model of PD

In this study, we investigated the cellular and behavioral effect of WT, S87A, and S87E $\alpha$-syn overexpression in the SNc of rats using a rAAV-delivery system. At expression levels equivalent to those of endogenous rat $\alpha$-syn, we observed that expression of human WT $\alpha$-syn induced $\alpha$-syn aggregate formation and a significant loss of dopaminergic neurons in the injected SNc ( $\sim 30 \%)$ compared to the noninjected and maxFP-Greeninjected side. Interestingly, the S87A mutant, which was designed to block phosphorylation, induced similar neuronal loss and aggregation properties as WT $\alpha$-syn. This observation is consistent with previous in vitro data from our group showing that this mutation does not alter the structural, membrane binding, or aggregation properties of $\alpha$-syn (Paleologou et al., 2010). In contrast, the phosphomimic S87E did not induce any significant neuronal loss compared to the noninjected or maxFP-Greeninjected side. The fact that WT $\alpha$-syn exhibited similar levels of aggregation and toxicity as S87A suggests that the extent of phosphorylation at S87 in dopamine (DA) neurons under these conditions is too low to impact the aggregation and toxicity of WT $\alpha$-syn. Indeed, using a semi-quantitative biochemical approach based on purified recombinant pS87-S129A $\alpha$-syn as reference material, the level of pS87 $\alpha$-syn was found to be $\sim 0.5-1 \%$ of total human $\alpha$-syn. On the basis of previous in vitro studies from our group, this level of pS87 is unlikely to influence the aggregation of WT $\alpha$-syn (Paleologou et al., 2008, 2010). It is noteworthy that WT $\alpha$-syn and both the S87A and S87E mutants showed similar levels of intraneuronal pS129 and exhibited similar patterns of nuclear and cytoplasmic localization, suggesting that 
A

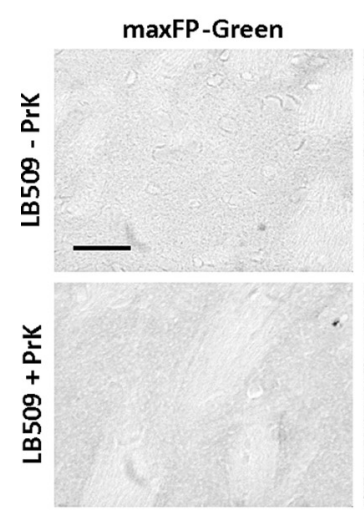

Syn WT

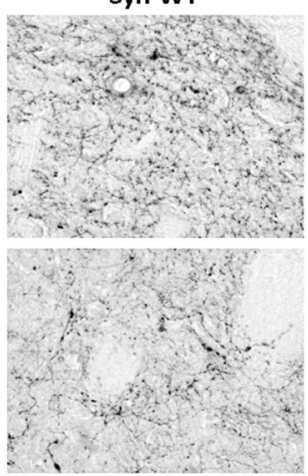

S87A

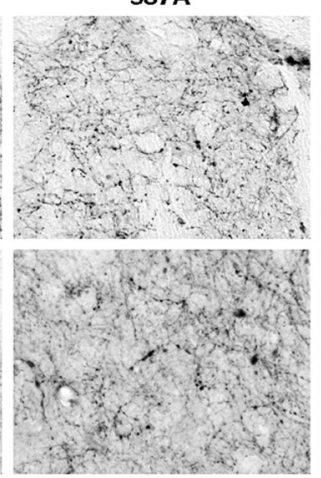

S87E

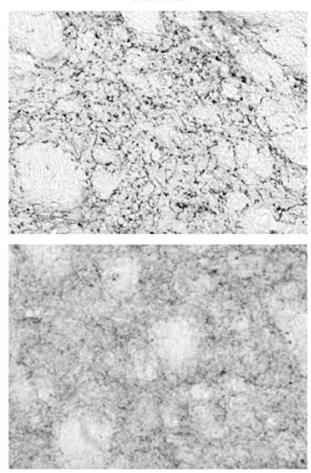

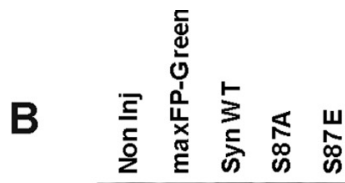

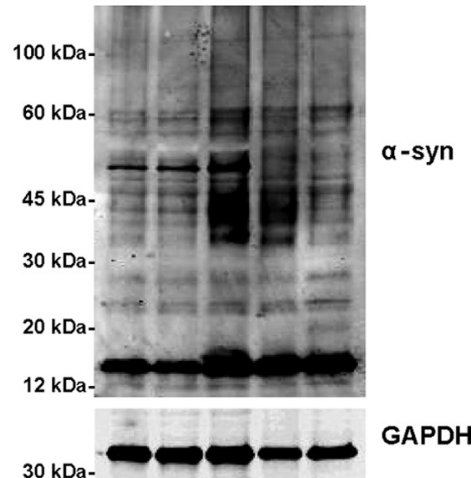

Figure 5. Syn WT and S87A form $\alpha$-syn aggregates. A, Photomicrographs illustrating the formation of Prk-aggregates in the striatum. The top row shows a high magnification of DAB immunostaining using anti-human $\alpha$-syn antibody (LB509) and reveals the presence of swollen and fragmented $\alpha$-syn-positive fibers. After digestion with PrK (bottom row), the staining showed a significant reduction in $\alpha$-syn signal in the striatum of S87E-injected animals. However, in the $\alpha$-syn WT- and S87A-injected animals, the staining revealed the presence of PrK-resistant $\alpha$-syn aggregates. Scale bar, $50 \mu \mathrm{m}$. B, Western blot of pooled samples extracted from injected midbrain and electrophoresed in 12\% SDS gel showed the formation high-molecular $\alpha$-syn species in the brains overexpressing Syn WT and S87A.

these S87 mutations do not affect $\alpha$-syn subcellular localization or phosphorylation at S129 within the infected neurons.

The overexpression of WT and S87A $\alpha$-syn, but not S87E, induces dopaminergic fiber pathology, which is characterized by swollen and dystrophic neurites. Interestingly, the dystrophic fibers, positive to ThS staining, colocalize with $\alpha$-syn deposits in the dorsolateral area of the striatum, suggesting that $\alpha$-syn aggregation could be responsible for the striatal fiber pathology formation. We observed significantly less dystrophic neurites in the S87E-injected animals, which correlate with the absence of $\alpha$-syn deposits.

In all cases, the extent of dopaminergic neuron loss correlated well with the induction of hemi-parkinsonian motor symptoms. Overexpression of human WT and S87A $\alpha$-syn induced hemiparkinsonian motor impairment, which was reflected by significantly reduced use of the contralateral forelimb. By contrast, no change in motor performance was observed in animals expressing S87E or maxFP-Green. It is worth noting that after induction of $25-30 \%$ of TH+ neuron loss, we observed a significant effect on the animal motor performance, suggesting that a moderate dopaminergic lesion is sufficient to induce a motor impairment in rats. This result is in agreement with a recent report by Koprich et al. (2010) demonstrating that overexpression of A53T $\alpha$-syn in the rat $\mathrm{SN}$ resulted in an impairment in striatal DA release and significant motor behavior deficits despite the fact that only a moderate dopaminergic lesion (28\%) was observed. Together, our results demonstrate that the serine-to-glutamic acid substitution reduces $\alpha$-syn-induced neuronal toxicity, fiber pathology, and motor deficits and suggest that phosphorylation at this site would block $\alpha$-syn toxicity in vivo.

\section{The $\alpha$-syn toxicity correlates with $\alpha$-syn aggregation and fibril formation in vivo}

Analysis of insoluble $\alpha$-syn aggregates in the striatum, which are a hallmark of PD and related disorders (Neumann et al., 2002), revealed that WT and S87A, but not S87E $\alpha$-syn, produces extensive PrK-resistant $\alpha$-syn deposits in the dorsolateral area of the striatum. Moreover, biochemical analysis of total protein extracted from the midbrain showed that WT and S87A form more high-molecular weight $\alpha$-syn oligomeric species compared to S87E mutant. These results are also consistent with previous in vitro data from our group demonstrating that WT and S87A $\alpha$-syn exhibit similar fibrillization properties, whereas the S87E mutant exhibit a marked reduction in oligomerization and fibril formation (Paleologou et al., 2010). S87 lies in the region, which is the hydrophobic and highly amyloidogenic part of the protein that is crucial for $\alpha$-syn aggregation and fibrillogenesis (Han et al., 1995; Iwai et al., 1995; El-Agnaf et al., 1998). Indeed, the introduction of a charged amino acid or a truncation within this region is known to inhibit $\alpha$-syn aggregation in vitro (El-Agnaf et al., 1998; Giasson et al., 2001) and in cell culture (Luk et al., 2009). Together, our studies demonstrate a direct correlation among $\alpha$-syn neurotoxicity, motor deficits, fiber pathology, and the extent of $\alpha$-syn aggregation and fibril formation in vivo and in vitro. Previous studies from two independent groups (Gorbatyuk et al., 2008; Azeredo da Silveira et al., 2009) also showed that there is a direct correlation between the extent of $\alpha$-syn fibrillization and loss of DA neurons in vivo. The S129A mutant, which forms fibrils more rapidly and to a greater extent than WT or the S129E mutant both in vitro (Paleologou et al., 2008) and in vivo (Gorbatyuk et al., 2008; Azeredo da Silveira et al., 2009), is more toxic than WT and S129E in the same model. Moreover, the S129A variant produced more $\alpha$-syn deposits in the midbrain compared to S129D (Azeredo da Silveira et al., 2009). These findings are consistent with recent studies demonstrating that the process of fibril formation plays a central role in the toxicity of amyloidforming proteins toxicity (Jan et al., 2008, 2010).

\section{Implications for PD therapies}

There has been a great interest in elucidating the role of $\alpha$-syn phosphorylation in the pathogenesis of $\mathrm{PD}$ with the hope that a better understanding of how this modification alters $\alpha$-syn aggregation and toxicity would lead to novel therapies based on targeting the activity of specific kinases or phosphatases. Together, the results presented within suggest that phosphorylation at $S 87$ is likely to protect against $\alpha$-syn-induced toxicity in vivo. The fact that WT and S87A exhibit similar aggregation and toxicity properties suggest that inhibiting the kinases involved in regulating 
$\alpha$-syn phosphorylation at $S 87$ is unlikely to show therapeutic benefits. Whether targeting phosphatases could constitute an alternative therapeutic strategy remains unclear and requires further studies to elucidate the role of $\$ 87$ phosphorylation in regulating other properties of $\alpha$-syn, including its degradation and interaction with other proteins and ligands. However, $\alpha$-syn mutations (S87E) that block fibrillization reduce its toxicity, while others (e.g., S129A) that increase fibrillization enhance $\alpha$-syn toxicity. These findings support a direct correlation between $\alpha$-syn fibrillization and neurotoxicity and suggest that lowering $\alpha$-syn levels and/or blocking its fibrillization are viable therapeutic strategies for the treatment of PD and related synucleinopathies.

\section{Notes}

Supplemental material for this article is available at http://lashuel-lab. epfl.ch/files/content/sites/nmnf/files/shared/Suppl\%20Materials.pdf. Evaluation of pS87 levels in vivo and assessment of S87 mutations effects on $\alpha$-syn phosphorylation at S129 and its subcellular localization. The pS87 level was evaluated using a semiquantitative approach based on the detection of known concentrations of recombinant pS87 and was found to be less than $1 \%$ of total $\alpha$-syn. Moreover, WT $\alpha$-syn and S87 mutants showed similar levels of intraneuronal pS129 and exhibited similar patterns of subcellular localization within the infected neurons. This material has not been peer reviewed.

\section{References}

Anderson JP, Walker DE, Goldstein JM, de Laat R, Banducci K, Caccavello RJ, Barbour R, Huang J, Kling K, Lee M, Diep L, Keim PS, Shen X, Chataway T, Schlossmacher MG, Seubert P, Schenk D, Sinha S, Gai WP, Chilcote TJ (2006) Phosphorylation of Ser-129 is the dominant pathological modification of alpha-synuclein in familial and sporadic Lewy body disease. J Biol Chem 281:29739-29752.

Azeredo da Silveira S, Schneider BL, Cifuentes-Diaz C, Sage D, Abbas-Terki T, Iwatsubo T, Unser M, Aebischer P (2009) Phosphorylation does not prompt, nor prevent, the formation of alpha-synuclein toxic species in a rat model of Parkinson's disease. Hum Mol Genet 18:872-887.

Baens M, Noels H, Broeckx V, Hagens S, Fevery S, Billiau AD, Vankelecom H, Marynen P (2006) The dark side of EGFP: defective polyubiquitination. PLoS One 1:e54.20.

Braak H, BraakE (2000) Pathoanatomy of Parkinson's disease. J Neurol 247 [Suppl 2]:3-10.

Chau KY, Ching HL, Schapira AH, Cooper JM (2009) Relationship between alpha synuclein phosphorylation, proteasomal inhibition and cell death: relevance to Parkinson's disease pathogenesis. J Neurochem 110:1005-1013.

Chen L, Feany MB (2005) Alpha-synuclein phosphorylation controls neurotoxicity and inclusion formation in a Drosophila model of Parkinson disease. Nat Neurosci 8:657-663.

Copeland M. XA, Turi T., Mouradian M. M., Braithwaite S. P., Martone R. L (2011) Parkinson's disease biomarker development: immunoprecipitation LC-MS/MS to quantitatively detect phosphorylated alpha-synuclein in human CSF. Paper presented at the 10th International Conference on Alzheimer's and Parkinson's Diseases, Barcelona, Spain, March.

Duda JE, Giasson BI, Gur TL, Montine TJ, Robertson D, Biaggioni I, Hurtig HI, Stern MB, Gollomp SM, Grossman M, Lee VM, Trojanowski JQ (2000) Immunohistochemical and biochemical studies demonstrate a distinct profile of alpha-synuclein permutations in multiple system atrophy. J Neuropathol Exp Neurol 59:830-841.

El-Agnaf OM, Jakes R, Curran MD, Middleton D, Ingenito R, Bianchi E, Pessi A, Neill D, Wallace A (1998) Aggregates from mutant and wild-type alpha-synuclein proteins and NAC peptide induce apoptotic cell death in human neuroblastoma cells by formation of beta-sheet and amyloid-like filaments. FEBS Lett 440:71-75.

Fournier M, Vitte J, Garrigue J, Langui D, Dullin JP, Saurini F, Hanoun N, Perez-Diaz F, Cornilleau F, Joubert C, Ardila-Osorio H, Traver S, Duchateau R, Goujet-Zalc C, Paleologou K, Lashuel HA, Haass C, Duyckaerts C, Cohen-Salmon C, Kahle PJ, et al. (2009) Parkin deficiency delays motor decline and disease manifestation in a mouse model of synucleinopathy. PLoS One 4:e6629.
Fujiwara H, Hasegawa M, Dohmae N, Kawashima A, Masliah E, Goldberg MS, Shen J, Takio K, Iwatsubo T (2002) alpha-Synuclein is phosphorylated in synucleinopathy lesions. Nat Cell Biol 4:160-164.

Giasson BI, Murray IV, Trojanowski JQ, Lee VM (2001) A hydrophobic stretch of 12 amino acid residues in the middle of alpha-synuclein is essential for filament assembly. J Biol Chem 276:2380-2386.

Gorbatyuk OS, Li S, Sullivan LF, Chen W, Kondrikova G, Manfredsson FP, Mandel RJ, Muzyczka N (2008) The phosphorylation state of Ser-129 in human alpha-synuclein determines neurodegeneration in a rat model of Parkinson disease. Proc Natl Acad Sci U S A 105:763-768.

Han H, Weinreb PH, Lansbury PT Jr (1995) The core Alzheimer's peptide NAC forms amyloid fibrils which seed and are seeded by beta-amyloid: is NAC a common trigger or target in neurodegenerative disease? Chem Biol 2:163-169.

Irizarry MC, Growdon W, Gomez-Isla T, Newell K, George JM, Clayton DF, Hyman BT (1998) Nigral and cortical Lewy bodies and dystrophic nigral neurites in Parkinson's disease and cortical Lewy body disease contain alpha-synuclein immunoreactivity. J Neuropathol Exp Neurol 57: 334-337.

Iwai A, Yoshimoto M, Masliah E, Saitoh T (1995) Non-A beta component of Alzheimer's disease amyloid (NAC) is amyloidogenic. Biochemistry 34:10139-10145.

Jakes R, Crowther RA, Lee VM, Trojanowski JQ, Iwatsubo T, Goedert M (1999) Epitope mapping of LB509, a monoclonal antibody directed against human alpha-synuclein. Neurosci Lett 269:13-16.

Jan A, Gokce O, Luthi-Carter R, Lashuel HA (2008) The ratio of monomeric to aggregated forms of Abeta 40 and Abeta 42 is an important determinant of amyloid-beta aggregation, fibrillogenesis, and toxicity. J Biol Chem 283:28176-28189.

Jan A, Hartley DM, Lashuel HA (2010) Preparation and characterization of toxic Abeta aggregates for structural and functional studies in Alzheimer's disease research. Nat Protoc 5:1186-1209.

Kahle PJ, Neumann M, Ozmen L, Müller V, Odoy S, Okamoto N, Jacobsen H, Iwatsubo T, Trojanowski JQ, Takahashi H, Wakabayashi K, Bogdanovic N, Riederer P, Kretzschmar HA, Haass C (2001) Selective insolubility of alpha-synuclein in human Lewy body diseases is recapitulated in a transgenic mouse model. Am J Pathol 159:2215-2225.

Kahle PJ, Neumann M, Ozmen L, Muller V, Jacobsen H, Spooren W, Fuss B, Mallon B, Macklin WB, Fujiwara H, Hasegawa M, Iwatsubo T, Kretzschmar HA, Haass C (2002) Hyperphosphorylation and insolubility of alpha-synuclein in transgenic mouse oligodendrocytes. EMBO Rep 3:583-588.

Khandelwal PJ, Dumanis SB, Feng LR, Maguire-Zeiss K, Rebeck G, Lashuel HA, Moussa CE (2010) Parkinson-related parkin reduces alphaSynuclein phosphorylation in a gene transfer model. Mol Neurodegener 5:47.

Kim EJ, Sung JY, Lee HJ, Rhim H, Hasegawa M, Iwatsubo T, Min do S, Kim J, Paik SR, Chung KC (2006) Dyrk1A phosphorylates alpha-synuclein and enhances intracellular inclusion formation. $J$ Biol Chem 281:33250-33257.

Kirik D, Georgievska B, Burger C, Winkler C, Muzyczka N, Mandel RJ, Bjorklund A (2002a) Reversal of motor impairments in parkinsonian rats by continuous intrastriatal delivery of L-dopa using rAAV-mediated gene transfer. Proc Natl Acad Sci U S A 99:4708-4713.

Kirik D, Rosenblad C, Burger C, Lundberg C, Johansen TE, Muzyczka N, Mandel RJ, Björklund A (2002b) Parkinson-like neurodegeneration induced by targeted overexpression of alpha-synuclein in the nigrostriatal system. J Neurosci 22:2780-2791.

Koprich JB, Johnston TH, Reyes MG, Sun X, Brotchie JM (2010) Expression of human A53T alpha-synuclein in the rat substantia nigra using a novel $\mathrm{AAV} 1 / 2$ vector produces a rapidly evolving pathology with protein aggregation, dystrophic neurite architecture and nigrostriatal degeneration with potential to model the pathology of Parkinson's disease. Mol Neurodegener 5:43.

Kragh CL, Lund LB, Febbraro F, Hansen HD, Gai WP, El-Agnaf O, RichterLandsberg C, Jensen PH (2009) \{alpha\}-Synuclein aggregation and Ser129 phosphorylation-dependent cell death in oligodendroglial cells. J Biol Chem 284:10211-10222.

Léger J, Kempf M, Lee G, Brandt R (1997) Conversion of serine to aspartate imitates phosphorylation-induced changes in the structure and function of microtubule-associated protein tau. J Biol Chem 272:8441-8446.

Lou H, Montoya SE, Alerte TN, Wang J, Wu J, Peng X, Hong CS, Friedrich EE, 
Mader SA, Pedersen CJ, Marcus BS, McCormack AL, Di Monte DA, Daubner SC, Perez RG (2010) Serine 129 phosphorylation reduces the ability of alpha-synuclein to regulate tyrosine hydroxylase and protein phosphatase 2A in vitro and in vivo. J Biol Chem 285:17648-17661.

Luk KC, Song C, O'Brien P, Stieber A, Branch JR, Brunden KR, Trojanowski JQ, Lee VM (2009) Exogenous alpha-synuclein fibrils seed the formation of Lewy body-like intracellular inclusions in cultured cells. Proc Natl Acad Sci U S A 106:20051-20056.

Machiya Y, Hara S, Arawaka S, Fukushima S, Sato H, Sakamoto M, Koyama S, Kato T (2010) Phosphorylated alpha-synuclein at Ser-129 is targeted to the proteasome pathway in a ubiquitin-independent manner. J Biol Chem 285:40732-40744.

McFarland MA, Ellis CE, Markey SP, Nussbaum RL (2008) Proteomics analysis identifies phosphorylation-dependent alpha-synuclein protein interactions. Mol Cell Proteomics 7:2123-2137.

McFarland NR, Fan Z, Xu K, Schwarzschild MA, Feany MB, Hyman BT, McLean PJ (2009) Alpha-synuclein S129 phosphorylation mutants do not alter nigrostriatal toxicity in a rat model of Parkinson disease. J Neuropathol Exp Neurol 68:515-524.

Neumann M, Kahle PJ, Giasson BI, Ozmen L, Borroni E, Spooren W, Müller V, Odoy S, Fujiwara H, Hasegawa M, Iwatsubo T, Trojanowski JQ, Kretzschmar HA, Haass C (2002) Misfolded proteinase K-resistant hyperphosphorylated alpha-synuclein in aged transgenic mice with locomotor deterioration and in human alpha-synucleinopathies. J Clin Invest 110:1429-1439.

Okochi M, Walter J, Koyama A, Nakajo S, Baba M, Iwatsubo T, Meijer L, Kahle PJ, Haass C (2000) Constitutive phosphorylation of the Parkinson's disease associated alpha-synuclein. J Biol Chem 275:390-397.

Oueslati A, Fournier M, Lashuel HA (2010) Role of post-translational modifications in modulating the structure, function and toxicity of alphasynuclein: implications for Parkinson's disease pathogenesis and therapies. Prog Brain Res 183:115-145.

Paleologou KE, Schmid AW, Rospigliosi CC, Kim HY, Lamberto GR, Fredenburg RA, Lansbury PT Jr, Fernandez CO, Eliezer D, Zweckstetter M, Lashuel HA (2008) Phosphorylation at Ser-129 but not the phosphomimics S129E/D inhibits the fibrillation of alpha-synuclein. J Biol Chem 283:16895-16905.
Paleologou KE, Oueslati A, Shakked G, Rospigliosi CC, Kim HY, Lamberto GR, Fernandez CO, Schmid A, Chegini F, Gai WP, Chiappe D, Moniatte M, Schneider BL, Aebischer P, Eliezer D, Zweckstetter M, Masliah E, Lashuel HA (2010) Phosphorylation at S87 is enhanced in synucleinopathies, inhibits alpha-synuclein oligomerization, and influences synuclein-membrane interactions. J Neurosci 30:3184-3198.

Schallert T, Fleming SM, Leasure JL, Tillerson JL, Bland ST (2000) CNS plasticity and assessment of forelimb sensorimotor outcome in unilateral rat models of stroke, cortical ablation, parkinsonism and spinal cord injury. Neuropharmacology 39:777-787.

Smith WW, Margolis RL, Li X, Troncoso JC, Lee MK, Dawson VL, Dawson TM, Iwatsubo T, Ross CA (2005) Alpha-synuclein phosphorylation enhances eosinophilic cytoplasmic inclusion formation in SH-SY5Y cells. J Neurosci 25:5544-5552.

Sugeno N, Takeda A, Hasegawa T, Kobayashi M, Kikuchi A, Mori F, Wakabayashi K, Itoyama Y (2008) Serine 129 phosphorylation of alphasynuclein induces unfolded protein response-mediated cell death. J Biol Chem 283:23179-23188.

Uéda K, Fukushima H, Masliah E, Xia Y, Iwai A, Yoshimoto M, Otero DA, Kondo J, Ihara Y, Saitoh T (1993) Molecular cloning of cDNA encoding an unrecognized component of amyloid in Alzheimer disease. Proc Nat Acad Sci U S A 90:11282-11286.

Waxman EA, Giasson BI (2008) Specificity and regulation of casein kinasemediated phosphorylation of alpha-synuclein. J Neuropathol Exp Neurol 67:402-416.

West MJ (1999) Stereological methods for estimating the total number of neurons and synapses: issues of precision and bias. Trends Neurosci 22:51-61.

Wu B, Liu Q, Duan C, Li Y, Yu S, Chan P, Uéda K, Yang H (2011) Phosphorylation of alpha-synuclein upregulates tyrosine hydroxylase activity in MN9D cells. Acta Histochem 113:32-35.

Zhou W, Milder JB, Freed CR (2008) Transgenic mice overexpressing tyrosine-to-cysteine mutant human alpha-synuclein: a progressive neurodegenerative model of diffuse Lewy body disease. J Biol Chem 283: 9863-9870. 